\title{
CARACTERIZAÇÃO FÍSICO-HÍDRICA DE UM PERFIL DE TERRA ROXA ESTRUTURADA LATOSSÓLICA PELO MÉTODO DO PERFIL INSTANTÂNEO ${ }^{(1)}$
}

\author{
A. C. S. da $\operatorname{COSTA}{ }^{(2)} \&$ P. L. LIBARDI ${ }^{(3)}$
}

\begin{abstract}
RESUMO
Em Terra Roxa Estruturada Latossólica do município de Piracicaba (SP), foram instalados tensiômetros a cada $15 \mathrm{~cm}$, em triplicata, a partir de $30 \mathrm{~cm}$ até à profundidade de $240 \mathrm{~cm}$, numa área de $25 \mathrm{~m}^{2}$, para medida do potencial mátrico $\left(\phi_{m}\right)$ e, indiretamente, por meio da curva de retenção de água, a umidade volumétrica $(\theta)$. $O$ método da umidade (Libardi et al., 1980) ajustou os dados de redistribuição de água ( $\theta$ vs $t)$ com coeficientes de determinação $\left(R^{2}\right)$ maiores que 0,98 . A hi pótese de gradiente do potencial hidráulico $\left(\delta \phi_{h} \delta z^{-1}\right)$ unitário foi verificada somente no horizonte $\mathrm{Bw}$. No horizonte $\mathrm{Bt}$, o gradiente do potencial hidráulico variou de 1 a $3,89 \mathrm{~m} \mathrm{~m}^{-1}$, o que determi nou valores de condutividade hidráulica maiores que os obtidos pelo método de Hillel et al. (1972). A umidade volumétrica na capacidade de campo $(\theta \mathrm{cc})$ variou de 0,45 a $0,33 \mathrm{~m}^{3} \mathrm{~m}^{-3}$, que corresponde a uma variação de potencial mátrico $\left(-\phi_{m}\right)$ de 0,0075 a $0,0014 \mathrm{MPa}$ e de conduti vidade hidráulica de 0,00068 a $0,00750 \mathrm{~cm} \mathrm{~h}^{-1}$. 0 horizonte $B t$, graças à estrutura em blocos bem desenvolvida, apresentou os maiores valores de densidade do solo e microporosidade, comparado aos do horizonte $\mathrm{Bw}$, que resultaram nas diferenças dos valores das características hídricas do perfil da TR.
\end{abstract}

Termos de indexação: morfologia, estrutura, porosidade, condutividade hidráulica, Bt, Bw.

(1) Parte da Tese de Mestrado do primeiro autor apresentada ao Curso de Pós-graduação em Solos e Nutrição de Plantas da Escola Superior de Agricultura "Luiz de Queiroz", Piracicaba (SP). Recebido para publicação em novembro de 1997 e aprovado em abril de 1999.

(2) Professor Adjunto do Departamento de Agronomia, Universidade Estadual de Maringá. Avenida Colombo, 5790. CEP 87020-900 Maringá (PR). Bolsista FAPESP/CNEN. Bolsista do CNPq.

(3) Professor Titular do Departamento de Física e Metereologia, ESALQ/USP. Bolsista do CNPq 
$\begin{aligned} \text { SUMMARY: } & \text { CHARACTERIZATION OF THE PHYSICAL AND HYDRAULIC } \\ & \text { PROPERTIES OF THE SOIL "TERRA ROXA ESTRUTURADA } \\ & \text { LATOSSÓLICA"BYTHE INSTANTANEOUS PROFILE METHOD }\end{aligned}$

Tensiometers were installed in a $25 \mathrm{~m}^{2}$ area plot, every $15 \mathrm{~cm}$, at depths varying from 30 to $240 \mathrm{~cm}$, with threerepetitions, to determinethematric potential $\left(\phi_{\mathrm{m}}\right)$ of a “Terra Roxa Estruturada Latossól ica" soil . I ts vol umetric water content was al so indi rectl y measured by using the soil water retention curve. The $\theta$-method (Libardi et al., 1980) fitted the soil redistribution profiles ( $\theta$ vs $\mathrm{t}$ ) with determination coefficients $\left(\mathrm{R}^{2}\right)$ greater than 0.98 . The hyphotesis of a unit hydraulic gradient was verified only in the Bw horizon. In theBt, the hydraulic gradient varied from 1.00 to $3.89 \mathrm{~m} \mathrm{~m}^{-1}$; thus the unsaturated hydraulic conductivity at these depths was greater than the values obtained by the Hille et al. (1972) procedure Thevol umetric soil water content at field capacity varied from 0.45 to $0.33 \mathrm{~m}^{3} \mathrm{~m}^{-3}$ that corresponds to a matric potential $\left(-\phi_{\mathrm{m}}\right)$ of 0.0075 to $0.0014 \mathrm{MPa}$ and an unsaturated hydraulic conductivity of 0.00068 to $0.00750 \mathrm{~cm} \mathrm{~h}^{-1}$. Increased bulk density and microporosity, as theresult of a block structureformation in theshallower Bt, compared to thedeeper Bw horizons, accounted for thevaluedifferences of thehydraulic properties of the TE soil.

Index terms: soil morphology, structure, porosity, hydraulic conductivity, Bw, Bt.

\section{NTRODUÇÃO}

Em regiões tropicais, é comum a ocorrência de associações de solos que apresentam transição do horizonte B-latossólico (Bw) para B-textural (Bt) (EMBRAPA, 1984). A gênese do horizonteBt revela como paradigma principal a argiluviação do horizonte A para o Bt. No entanto, para Moniz \& Buol (1982), a transformação $B w \Rightarrow B t$ ocorre por adensamento do Bt a partir do Bw em virtude das "deformações plásticas induzidas pelos ciclos de umedecimento e de secagem" (Moniz, 1996) associadas ao fluxo lateral e basal da solução do solo.

Este paradigma nos estudos de gênese utiliza fundamentos da mecânica do solo etem si do val idado no campo por meio da avaliação das características morfológicas e físicas do solo, tais como: densidade, porosidade e variação no teor de água (Moniz et al., 1994). Não há, no entanto, número significativo de estudos que avaliem o comportamento hídrico do solo ondeneleocorrea transi ção Bt para oBw. Além disso, a relação umidade volumétrica - condutividade hidráulica $(K(\theta))$, a curva de retenção de água $\left(\theta\right.$ vs $\left.\phi_{m}\right)$, a umidade do solo na capaci dade de campo $(\theta c c)$ e no ponto de murchamento permanente $\left(\theta_{\mathrm{PMP}}\right)$ são determinações importantes no cál culo do bal anço hídrico das culturas agrícol as (Saunders et al., 1978; Reichardt, 1985), bem como na resolução de problemas como erosão do solo e contaminação do lençol freático.

O perfil instantâneo (Hillel et al., 1972) éum dos métodos mais utilizados na determinação da condutividade hidráulica do sol o não saturado pela sua simplicidade experimental ematemática. Outros autores têm aplicado, com sucesso, este método em diferentes condições de solo (Freire, 1979; Reichardt \& Libardi, 1974). Baker et al. (1974) observaram que o método apresenta limitações quando aplicado em solos de relevo ondulado e recomendaram isolar a parcela em que será feita a determinação, para que não ocorra fluxo lateral de água, principal menteem solos que apresentam horizontes geneticamente densos (Bt) ou compactados em decorrência de seu manejo inadequado. I sto dificultaria a saturação do perfil em camadas mais profundas, não sendo obtidos os valores de condutividade hidráulica para teores de umidade próximos à saturação. Por este método, é possível ainda obter o val or da umidade do solo na capacidade de campo $(\theta c c)$, que constitui o limite superior do teor de água para as plantas e básico no cálculo do teor de água disponível do solo.

As características do solo, como textura, densidade, matéria orgânica, macroporosidade e microporosidade, têm influência na retenção e movimento de água no solo. Solos aparentemente homogêneos quanto à textura e mi neralogia, quando apresentam horizontes geneticamente mais (Bt) ou menos (Bw) densos revelam comportamentos diferentes em relação à retenção e movimento da água (Zimback \& Carvalho, 1996).

O objetivo deste trabal ho foi avaliar a influência das características morfológicas dos horizontes Bt e Bw num perfil de Terra Roxa Estruturada Latossólica nas suas características físicas e hídricas. 


\section{MATE RIAL E MÉTODOS}

O sol o estudado pertence ao Grande Grupo Terra Roxa Estruturada (TR), série "Luiz de Queiroz", localizado em área próxima à estação meteorológica da Escola Superior deAgricultura "Luiz de Queiroz", Piracicaba (SP). Foi delimitada uma parcela experimental de $25 \mathrm{~m}^{2}(5 \times 5 \mathrm{~m})$, tendo sido instalados tensiômetros com manômetro demercúrio para determinação do potencial mátrico $\left(\phi_{\mathrm{m}}\right)$ a cada $15 \mathrm{~cm}$ deprofundidade, em triplicata, a partir de $30 \mathrm{~cm}$ da superfície do solo até à profundidade de $240 \mathrm{~cm}$, num total de 45 instrumentos.

Os valores de umidade volumétrica $(\theta)$ foram obtidos indiretamente por meio da curva de retenção $\left(\theta\right.$ vs $\left.\phi_{m}\right)$. Em área próxima, foi aberta uma trincheira para descrição morfológica do perfil do solo (Lemos \& Santos, 1984). Retiraram-se amostras com estrutura indeformada, para determinação da curva de retenção e da densidade do solo, e amostras com estrutura deformada, para determinação das suas características químicas efísicas. A densi dade do solo e a curva de retenção de água foram determinadas nas amostras de estrutura indeformada, utilizando cilindros de Köpeck de $50 \mathrm{~cm}^{3}$ com bordos cortantes nas profundidades de instalação dos tensiômetros. A curva de retenção de água foi determinada nas tensões de 0,0001 a 0,015 MPa, em funil de placa porosa, e de 0,033 a 1,5 MPa, na câmara de pressão de Richards. A análise granulométrica foi efetuada pelo método da pipeta (EMBRAPA, 1979) em amostras de sol o retiradas aolado das utilizadas na determinação da curva de retenção da água. A porosidade total $(\mathrm{P})$ foi calculada pelo valor da umidade volumétrica na tensão de 0,0001 MPa. A microporosidade (Mi) foi considerada igual ao teor de água retido na tensão de $0,006 \mathrm{MPa}$ e a macroporosidade ( $\mathrm{Ma}$ ) foi obtida pela diferença entre a porosidade total e a microporosidade. A umidade volumétrica do solo no ponto de murchamento permanente $\left(\theta_{\mathrm{PMP}}\right)$ foi considerada igual a umidade volumétrica na tensão de 1,5 MPa.

A condutividadehidráulica do sol o foi determinada no campo pel o método do perfil instantâneo, utilizando os procedimentos de Hillel et al . (1972) e o método- $\theta$ de Libardi et al. (1980). O método de Hillel et al. (1972) diferencia-se do método- $\theta$ de Libardi et al. (1980) por determinar a variação do potencial hidráulico com a distância $\left(\delta \phi_{\mathrm{h}} \delta \mathrm{z}^{-1}\right)$ e da umidade em função da profundidade ( $\delta \theta \delta z^{-1}$ ) por meio de métodos gráficos que, embora relativamentesimples, são muito demorados e trabal hosos. O método- $\theta$ de Libardi et al. (1980) possui não sóa diferença básica de considerar unitário o gradiente do potencial hidráulico $\left(\delta \phi_{\mathrm{h}} \delta \mathrm{z}^{-1}=1\right)$, mas também, analiticamente, a variação da umidade com o tempo no processo de drenagem interna do perfil.

Pelo método do perfil instantâneo, após a instalação dos tensiômetros, a área de $25 \mathrm{~m}^{2}$ foi cercada por quatro fol has de flandres enterradas $15 \mathrm{~cm}$ no solo e, em seguida, adicionou-se água, mantendo-se uma lâmina de $5 \mathrm{~cm}$ até atingir a condição de equilíbrio dinâmico observado pela leitura constante em todos os tensiômetros. Após, cessou-se o fornecimento de água e cobriu-se a área experimental com uma Iona plástica, a fim de prevenir a evaporação e a entrada de água através da superfície do solo. A drenagem interna do perfil do solo foi verificada por medidas periódicas dos tensiômetros, necessárias ao cál culo do potencial hidráulico (Hillel et al., 1972). As leituras dos tensiômetros foram efetuadas a partir da cobertura do solo ( $\mathrm{t}=0 \mathrm{~h}$ ) por um período de 23 dias. Pelo método- $\theta$ (Libardi et al., 1980), a condutividade hidráulica é uma função exponencial da umidade volumétrica:

$$
K(\theta)=K_{o} \text { e } \gamma(\theta-\theta 0)
$$

em que $K_{\circ}$ e $\theta_{0}$ são a condutividade hidráulica e a umidade volumétrica em condições de equilíbrio dinâmico e $\gamma$ é um coeficiente obtido no processo de redistribuição da água. A equação analítica que descreve o processo de drenagem interna é:

$$
\theta=\theta_{0}-1 / \gamma\left\{n\left[1+\left(\gamma K_{0} t / \alpha z\right)\right]\right\}
$$

em que $\alpha$ é uma constante que depende da profundidade $z$ e do tempo $t$. A umidade do solo na capacidade de campo ( $\theta \mathrm{cc}$ ) foi determinada pela média das quatro últimas observações $(250,353,443$, e 518 h), visto que, após oito dias, o gradiente do potencial hidráulico praticamente não variou. Maiores detalhes de ambos os métodos podem ser encontrados em Libardi (1995).

\section{RESULTADOS E DISCUSSÃO}

A descrição morfológica do perfil do solo (Quadro 1) apresentou uma seqüência de horizontes Ap, Bt e Bw, identificáveis no campo pela variação em profundidade da cor, textura, estrutura, consistência e presença de cerosidade no Bt, inexistenteno horizonte Bw (Lemos \& Santos, 1984).

Algumas características da Terra Roxa Estruturada Latossólica (Quadro 2) revelaram alto conteúdo de argila, pertencendo o solo à classe textural argila, nas profundidades de $105-240 \mathrm{~cm}$, e à classe muito argilosa, nas profundidades de 30$90 \mathrm{~cm}$. Os maiores teores de argila ocorreram nos horizontes superficiais até à base do horizonte Bt (90 cm, Quadro 2), decrescendo no horizonte Bw. Esse acréscimo no teor de argila foi devido não somente à iluviação de material dos horizontes superficiais observado pela cer osidade, mas também à formação de argila in situ, resultando num maior adensamento dos agregados e na formação da estrutura em blocos típica do Bt (M oniz et al., 1982; Moniz, 1996). Isto pode ser confirmado pelos 
Quadro 1. Sumário da descrição morfológica da Terra Roxa Estruturada Latossólica

\begin{tabular}{|c|c|c|c|c|c|}
\hline Horizonte & Prof. & Cor úmida & Textura ${ }^{(1)}$ & Estrutura/ cerosidade ${ }^{(2)}$ & Consistência $^{(3)}$ \\
\hline & $\mathrm{cm}$ & & & & \\
\hline Ap & $0-21$ & $2,5 Y R 4 / 4$ & Argila & Pequeno bloco subangular forte e grande granular forte & Duro, firme; plástico e pegajoso \\
\hline Bt1 & $21-60$ & $2,5 Y R 3 / 2$ & Muito argiloso & $\begin{array}{l}\text { Média e grande bloco subangular forte; cerosidade forte e } \\
\text { abundante }\end{array}$ & Duro, firme; plástico e pegajoso \\
\hline Bt2 & $60-90$ & $10,0 \mathrm{R} 3 / 6$ & Muito argiloso & $\begin{array}{l}\text { Grande bloco subangular moderado que se desfaz, forte } \\
\text { muito pequeno granular; cerosidade fraca e pouca }\end{array}$ & $\begin{array}{l}\text { Duro, firme; muito plástico e } \\
\text { muito pegajoso }\end{array}$ \\
\hline Bw & $90-240+$ & $2,5 Y R 5 / 6$ & Muito argiloso & $\begin{array}{l}\text { Fraca, grande bloco subangular que se desfaz, forte muito } \\
\text { pequena granular }\end{array}$ & $\begin{array}{l}\text { Ligeiramente duro, friável; } \\
\text { muito plástico e muito pegajoso }\end{array}$ \\
\hline
\end{tabular}

Quadro 2. Teor de carbono orgânico (C), areia, silte e argila, nas diversas profundidades da Terra Roxa Estruturada Latossólica

\begin{tabular}{|c|c|c|c|c|}
\hline Profundidade & C & Areia & Silte & Argila \\
\hline $\mathrm{cm}$ & \multicolumn{4}{|c|}{$\mathrm{kg} \mathrm{kg}^{-1}$} \\
\hline 30 & 6,6 & 0,24 & 0,15 & 0,61 \\
\hline 45 & 4,2 & 0,21 & 0,15 & 0,64 \\
\hline 60 & 2,7 & 0,22 & 0,15 & 0,63 \\
\hline 75 & 3,3 & 0,22 & 0,16 & 0,62 \\
\hline 90 & 2,4 & 0,22 & 0,16 & 0,62 \\
\hline 105 & 3,0 & 0,24 & 0,17 & 0,59 \\
\hline 120 & 2,4 & 0,24 & 0,18 & 0,58 \\
\hline 135 & 2,1 & 0,25 & 0,17 & 0,58 \\
\hline 150 & 1,2 & 0,25 & 0,19 & 0,56 \\
\hline 165 & 1,5 & 0,25 & 0,19 & 0,57 \\
\hline 180 & 0,6 & 0,26 & 0,19 & 0,55 \\
\hline 195 & 1,5 & 0,26 & 0,18 & 0,56 \\
\hline 210 & 0,9 & 0,26 & 0,16 & 0,58 \\
\hline 225 & 0,3 & 0,24 & 0,18 & 0,58 \\
\hline 240 & 0,3 & 0,23 & 0,18 & 0,59 \\
\hline
\end{tabular}

resultados de densidade do solo que variaram de 1,43 a $1,21 \mathrm{~kg} \mathrm{~m}^{-3}$ (Figura 1 ), sendo os maiores valores encontrados nas camadas próximas à superfície onde se encontra o horizonte Bt (Moniz \& Buol, 1982). A partir do horizonte Bt, percebeu-se um decréscimo na densidade e cerosi dade, bem como o aparecimento de uma estrutura granular muito pequena forte, típica do horizonte Bw.

A distribuição da quantidade de poros totais e microporos não apresentou nenhuma correlação com os valores de densidade do solo (Figura la). No entanto, verificou-se uma correlação positiva $\left(R^{2}=\right.$ 0,7294 ) entre a percentagem relativa de microporos e os valores de densidade do solo (Figura 1b). Os maiores teores de argila e de densidade do solo dos horizontes (Bt) determinaram uma maior

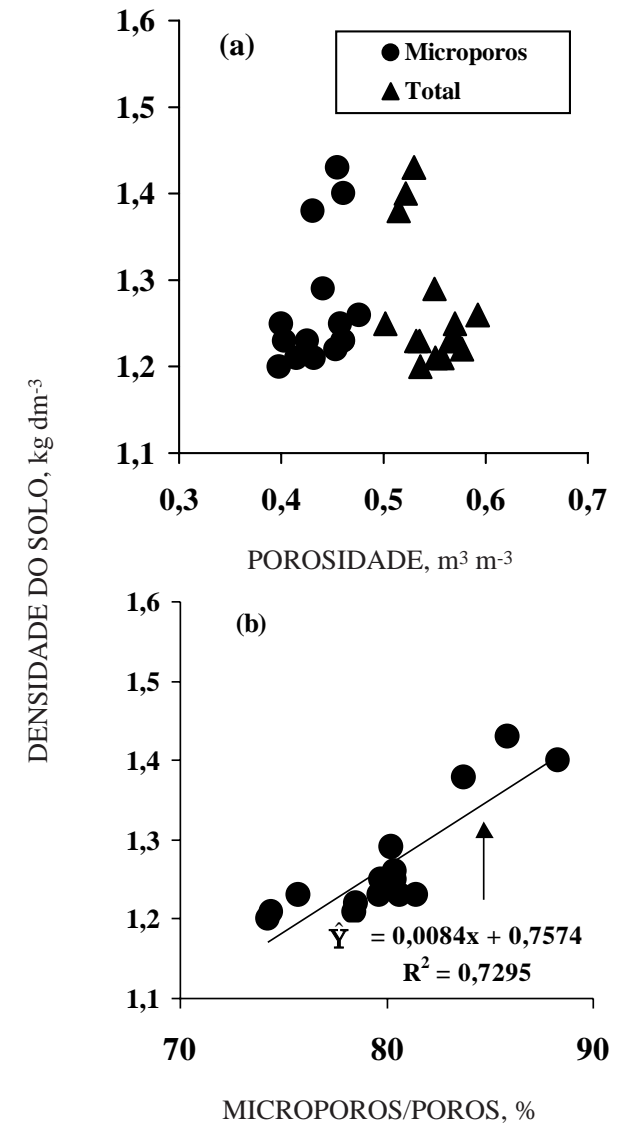

Figura 1. Porosidade total, microporosidade absoluta $\left(1 \mathrm{a}, \mathrm{m}^{3} \mathrm{~m}^{-3}\right.$ ) e microporosidade relativa (1b, \%) nas diferentes profundidades.

microporosidade, enquanto o horizonte Bw apresentou os maiores val ores de porosidade total e macroporos (Figura 1). Os valores de porosidade total, macro emicroporosidade obtidos na TR foram da mesma ordem de valores encontrados em outros trabalhos (Reichardt \& Libardi, 1974; Freire, 1979). 
Os valores de umidade volumétrica obtidos para os respectivos potenciais mátricos determinaram, numa escala semilogarítmica, curvas de retenção de água que refletiram a distribuição de poros nos respectivos horizontes do solo. As curvas de retenção representativas do horizonte $\mathrm{Bt}(45,60$ e $75 \mathrm{~cm})$, entre os potenciais mátricos de 0,01 e 1,5 MPa (microporos), formaram, na escala semilogarítmica, retas praticamente paralelas, evidenciando distribuição semelhante de tamanho de poros. O horizonte Bw $(150 \mathrm{~cm})$ apresentou comportamento semel hante somente nos potenciais mátricos maiores (0,03-1,5 MPa; Figura 2). Os valores de porosidade entre as tensões de 0,01 e 0,0001 MPa, em que predominam os macroporos, foram maiores nos horizontes superficiais que em profundidade. $\mathrm{O}$ horizonte Bw apresentou maior retenção de água em tensões menores que 0,001 MPa (macroporos) e menor retenção em tensões maiores que 0,01 MPa (microporos). A quantidade de poros observada entre as tensões de 0,0001 e 0,015 MPa (Figura 2) foi de $0,0938 \mathrm{~m}^{3} \mathrm{~m}^{-3}$ na profundidade de $45 \mathrm{~cm}$ (Bt); menos da metade do valor obtido para a profundidade de $150 \mathrm{~cm}(\mathrm{Bw})$, que foi de $0,2112 \mathrm{~m}^{3} \mathrm{~m}^{-3}$. O horizonte Bw (90-240 cm), embora pertença à classe textural muito argilosa, revel ou características hídricas muito semelhantes às dos sol os arenosos (U ehara \& Gillman, 1981), decorrentes de seus baixos valores de densidade e microporos (Figuras 1 e 2). Tal comportamento do Bw pôde ainda ser observado, comparando-se a sua menor retenção de água na $\theta_{\text {PMP }}$, cuja diferença no valor absoluto entre as camadas de 45 e $150 \mathrm{~cm}$ (Figura 2) foi de 0,05 $\mathrm{m}^{3} \mathrm{~m}^{-3}$.

Os valores da umidade volumétrica do solo obtidos no tempo inicial $\left(\theta_{0}\right)$ do processo de redistribuição de água (Figura 3) foram inferiores aos observados na tensão de $0,0001 \mathrm{MPa}$ nas respectivas profundidades (Figura 2 ), pel o fato de 0 horizonte Bt apresentar maior densidade e microporosi dade que impedem o movimento de água subsuperficial mente em quantidades necessárias à saturação do horizonte Bw (Souza et al., 1979). Outros autores encontraram semelhantes dificuldades (Saunders et al., 1978; Freire, 1979), o que restringe a faixa de umidade volumétrica $(\theta)$ para determinação da função $\mathrm{K}(\theta)$ em perfis de solos que apresentam anisotropia (Baker et al., 1974) em relação à suas propriedades físicas associadas a variações na sua morfologia.

A redistribuição da água foi maior nas camadas subsuperficiais (Bw) quando comparadas à das camadas superficiais (Bt). As variações no teor de água no processo de redistribuição de água foram maiores nos primeiros oito dias, tornando-se praticamente constantes depois (Figura 3). Considerando a pequena variação na composição granulométrica (Quadro 2) e mineralógica (Demattê \& Marconi, 1991; Demattê et al., 1994) do perfil do solo, pode-seafirmar queas variações noteor deágua estão mais diretamente ligadas à distribuição dos poros associados às diferenças nas características morfológicas dos horizontes Bt e Bw discutidas anteriormente. Percebeu-se um excelenteajuste dos dados experimentais do processo de redistribução de água e o model o matemático do método- $\theta$ de Libardi et al. (1980) que apresentaram (Quadro 3) altos coeficientes de determinação $\left(R^{2}>0,98\right)$.

Os valores de condutividade hidráulica do solo não saturado obtidos pelo método- $\theta$ (Libardi et al . 1980) com a suposição de gradiente do potencial hidráulico unitário $\left(\delta \phi_{\mathrm{h}} \delta z^{-1}=1 \mathrm{~m} \mathrm{~m}^{-1}\right)$ apresentaram tendência semel hante aos determi nados pelo método de Hillel et al. (1972) (Figura 4). No entanto, tal suposição determinou uma superestimativa nos valores de condutividade hidráulica entre as camadas de $45-120 \mathrm{~cm}$, pois o gradiente do potencial hidráulico foi maior queum (Figura 5), observandose val ores de 3,89 $\mathrm{m} \mathrm{m}^{-1}$ na profundidade de $45 \mathrm{~cm}$.

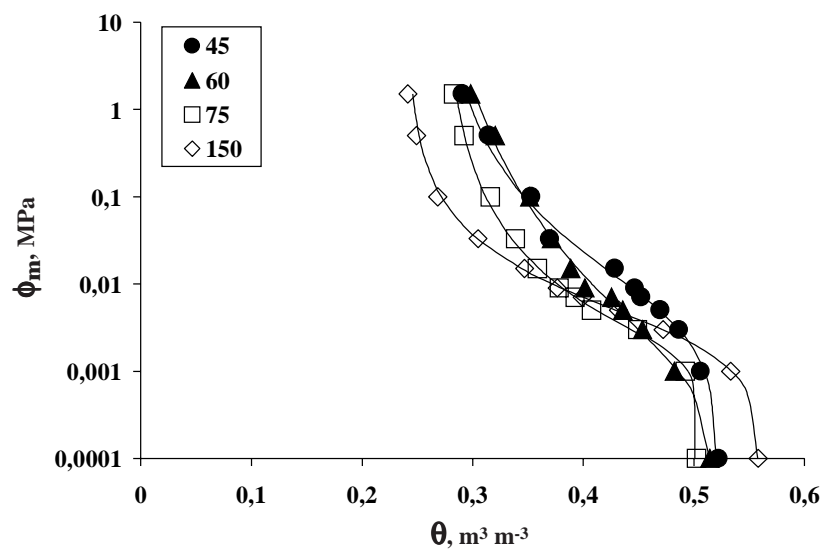

Figura 2. Potencial mátrico $\left(\phi_{m}\right)$ e umidade volumétrica $(\theta)$ da curva de retenção de água dos horizonte Bt $(45,60,75 \mathrm{~cm})$ e Bw $(150 \mathrm{~cm})$.

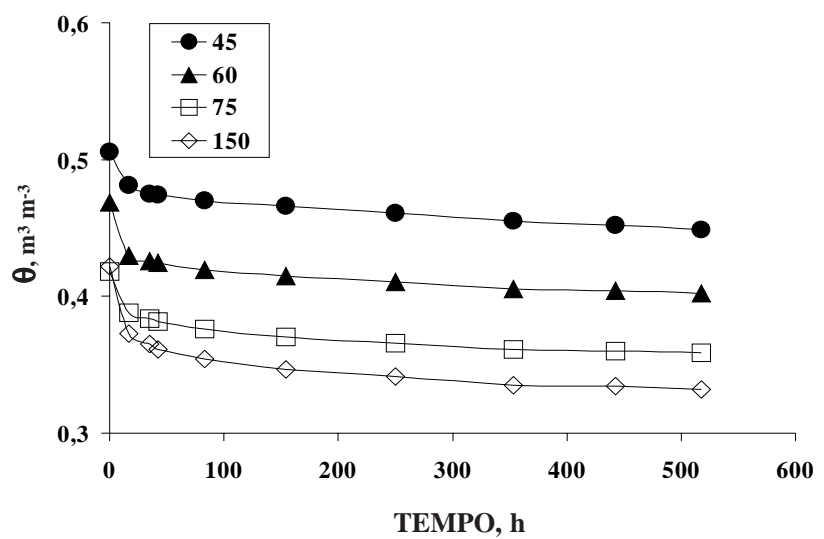

Figura 3. Umidade volumétrica $(\theta)$ em função do tempo no processo de redistribuição de água do horizonte Bt $(45,60$, 75 cm) e Bw (150). 
Quadro 3. Parâmetros $\left(K_{0}, \gamma, \theta_{0}, \alpha\right)$ obtidos no processo de redistribuição da água nas diversas profundidades para o método- $\theta$ (Libardi et al., 1980)

\begin{tabular}{cccccc}
\hline Profundidade & $\mathbf{K}_{\mathbf{0}}$ & $\boldsymbol{\gamma}$ & $\boldsymbol{\theta}$ & $\boldsymbol{\alpha}$ & $\mathbf{R}^{\mathbf{2}}$ \\
\hline $\mathrm{cm}$ & $\mathrm{cm} \mathrm{h}-1$ & & $\mathrm{~m}^{3} \mathrm{~m}^{-3}$ & & \\
30 & 0,43450 & 107,46419 & 0,5050 & 1 & 0,98621 \\
45 & 0,46348 & 112,03416 & 0,5058 & 0,98996 & 0,98562 \\
60 & 0,99977 & 120,51732 & 0,4685 & 1,08471 & 0,98944 \\
75 & 1,13526 & 112,46883 & 0,4178 & 1,00917 & 0,99261 \\
90 & 3,12973 & 81,96021 & 0,4835 & 0,77651 & 0,99473 \\
105 & 4,05736 & 89,48702 & 0,4086 & 0,86988 & 0,99584 \\
120 & 6,81853 & 86,27106 & 0,4485 & 0,85841 & 0,99630 \\
135 & 10,51103 & 79,17732 & 0,4866 & 0,80929 & 0,99661 \\
150 & 13,10703 & 83,26083 & 0,4317 & 0,86535 & 0,99711 \\
165 & 12,14771 & 64,72109 & 0,5216 & 0,69543 & 0,99753 \\
180 & 11,95091 & 62,25807 & 0,4708 & 0,68972 & 0,99742 \\
195 & 11,36850 & 60,61545 & 0,4853 & 0,69042 & 0,99792 \\
210 & 11,06888 & 59,07993 & 0,4696 & 0,69027 & 0,99784 \\
225 & 10,71374 & 59,26518 & 0,4689 & 0,70801 & 0,99780 \\
240 & 11,31309 & 62,39568 & 0,4557 & 0,75829 & 0,99793 \\
\hline
\end{tabular}

Saunders et al. (1978), em condições experimentais semel hantes, obtiveram valores de gradiente do potencial total numa profundidade de $120 \mathrm{~cm}$ para um solo Terra Roxa Estruturada que variaram de 2,02-2,82 m m-1. O horizonte Bw (150 cm, Figura 4) apresentou valores de condutividade hidráulica não saturada muito semelhantes nos dois métodos em razão da existência de um gradiente do potencial hidráulico próximo à unidade (150cm, Figura 5).

A variação do gradiente do potencial hidráulico foi maior nos horizontes superficiais (Bt), considerando as variações nas suas características morfológicas (Quadro 1), físicas (Quadro 2, Figuras 1 e 2), quando comparadas com as do horizonte Bw. Por exemplo, para calcular o gradiente do potencial hidráulico na camada de $60 \mathrm{~cm}$, considerou-se a variação no potencial hidráulico entre as camadas de 45 e $75 \mathrm{~cm}$. Dos componentes do potencial hidráulico, somente o potencial mátrico influiu na energia de retenção da água pelo solo, pois o componente potencial gravitacional $\left(\delta \phi_{\mathrm{g}}\right)$ age independente do meio. Portanto, os valores do gradiente do potencial hidráulico só podem ser atribuídos às diferenças entre o potencial mátrico determinados nos diferentes tempos de redistribuição nas profundidades consideradas. A partir da profundidade de $75 \mathrm{~cm}$, o cálculo do gradiente do potencial hidráulico foi feito somente entre camadas contidas no horizonte $\mathrm{Bw}$, que apresentaram muita semelhança nas suas características morfológicas (Quadro 1), físicas (Quadro 2) e hídricas (Figuras 2 e 6). A variaçãonos valores do gradiente do potencial hidráulico pode, portanto, ser utilizada como um indicativo da presença de anisotropia vertical entre os horizontes de um perfil de solo. Os resultados obtidos demonstraram a existência de restrições à hipótese de gradiente do potencial hidráulico unitário em sol os com adensamento natural (Bt), compactação ou com características morfológi cas heterogêneas no perfil do solo (Vauclin et al., 1982), mas não inviabilizaram sua utilização em virtude da inerente variabilidade espacial dessas características no solo (Lauren et al., 1988).

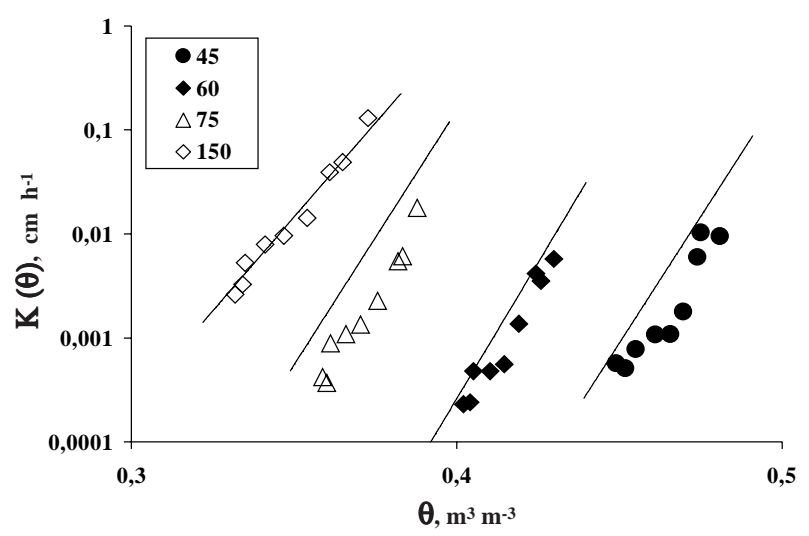

Figura 4. Condutividade hidráulica insaturada do horizonte Bt $(45,60,75 \mathrm{~cm})$ e Bw $(150 \mathrm{~cm})$ obtidos pelo método de Hillel et al. (1972) (símbolos) e Libardi et al. (1980) (retas). 


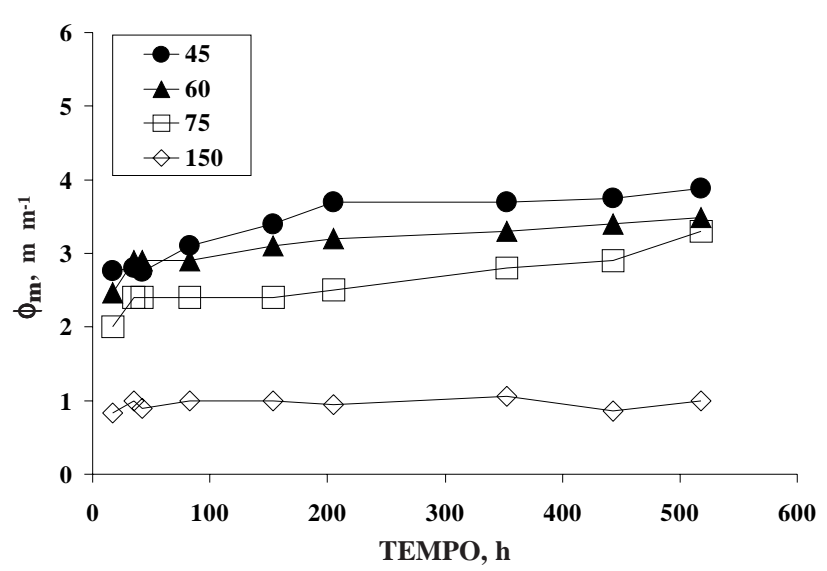

Figura 5. Gradiente do potencial hidráulico $\left(\delta \phi_{h} \delta z^{-1}\right)$ em função do tempo no processo de drenagem interna do horizonte $B t(45,60$, $75 \mathrm{~cm})$ e Bw $(150 \mathrm{~cm})$.

A condutividade hidráulica em condições de equilíbrio dinâmico $\left(\mathrm{K}_{0}\right)$ aumentou em profundidade até à camada de $150 \mathrm{~cm}$ (Quadro 3), mantendo-se então praticamente constante, indicando a maior velocidade deinfiltração nas camadas correspondentes ao horizonte Bw por causa de sua maior macroporosidade (Quadro 2). Zimback \& Carvalho (1996) observaram fato semel hante em Podzólicos e concluíram que as características físicas e hídricas dos solos deveriam ser avaliadas nos horizontes subsuperficiais (Bt) por serem estes os controladores do fluxo de água. Os val ores de $K_{0}$ observados foram inferiores aos de Freire (1979), principalmente nas camadas superficiais, diminuindo as diferenças nas camadas mais profundas, que apresentaram maior semelhança morfológica com o perfil do Latossolo Roxo.
A condutividade hidráulica, em qualquer valor de umidade volumétrica, foi sempre menor nas profundidades correspondentes ao horizonte Bt que noBw. Os valores baixos de condutividade hidráulica no horizonte Bt determinaram o aparecimento de zonas de acumulação de água, capazes de favorecer o deslocamento de minerais de argila dispersos, que, depositados nos poros do solo, aumentaram o teor de argila, microporosidade, criando condições para a formação do horizonte Bt por adensamento do Bw (Moniz et al., 1982; Moniz \& Buol, 1982; Moniz, 1996).

A umidade do solo na capaci dade de campo ( $\theta c c)$ diminuiu em profundidade em decorrência da maior macroporosidade dessas camadas e atingiu o máximo de $0,45 \mathrm{~m}^{3} \mathrm{~m}^{-3}$ na profundidade de $45 \mathrm{~cm}$, onde também foi determinado o maior valor de microporosidade, i. é, na profundidade de máxima expressão do horizonte Bt. Saunders et al. (1978), em condições semel hantes, só que utilizando sonda de nêutrons para medida da umidade volumétrica, encontraram val ores mais baixos de $\theta$ cc nas camadas de 30,60 e $90 \mathrm{~cm}$ e valor semel hante para profundidade de $120 \mathrm{~cm}$, refletindo as variações nas medidas desses parâmetros, quando diferentes instrumentos for am utilizados.

Freire (1979) observou num Latossolo Roxo que a $\theta c c$ variou de 0,33-0,37 $\mathrm{m}^{3} \mathrm{~m}^{-3}$, valores semel hantes aos observados nas camadas mais profundas (105$240 \mathrm{~cm}$ ) da TR. Os valores de potencial mátrico na capacidade de campo variaram de 0,0075 a $0,0014 \mathrm{MPa}$ (Figura 6), aumentandoem profundidade. Tais val ores, determinados in situ, representaram o potencial mátrico nas condições de capacidade de campo e foram próximos aos valores de 0,01 MPa, utilizados em sol os tropicais (Arruda et al., 1987), e maiores que 0,033 $\mathrm{MPa}$, utilizados em solos de regiões temperadas (Haise et al., 1955). Freire(1979) obteve valores de potencial mátrico na capacidade
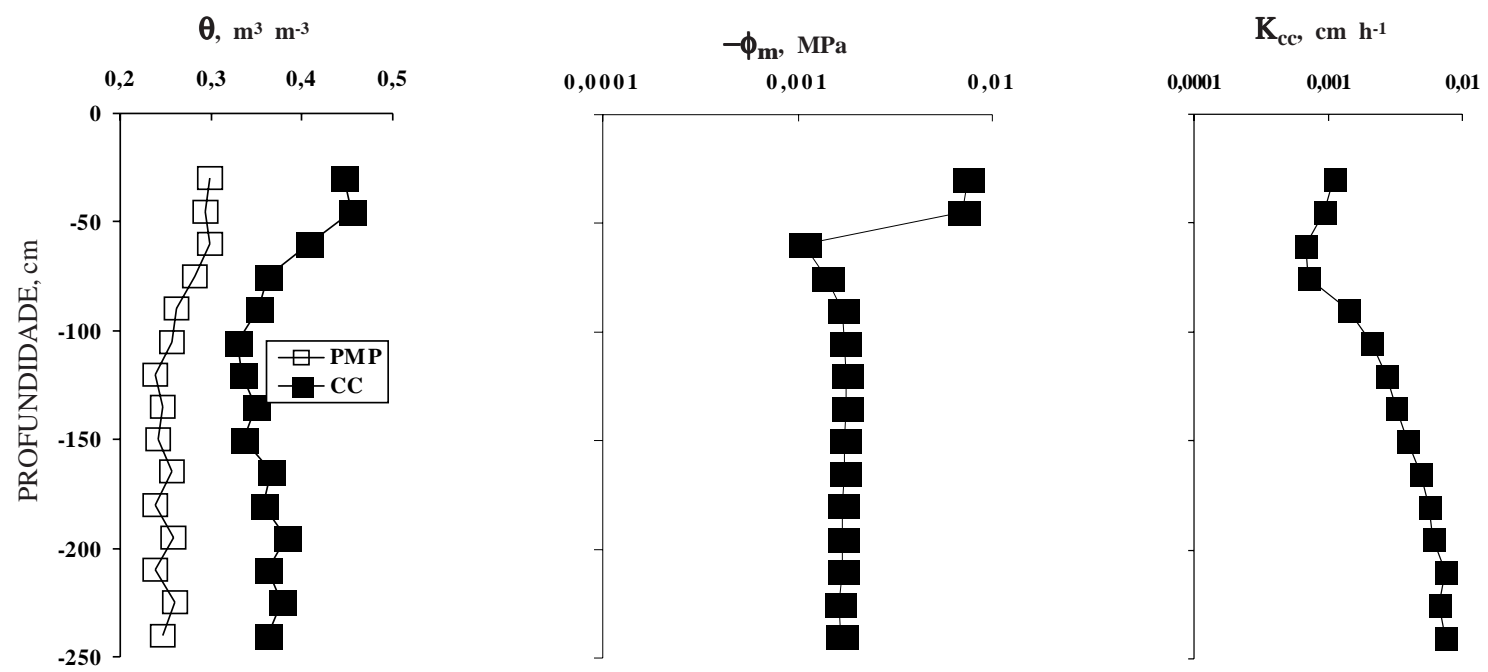

Figura 6. Umidade volumétrica $(\theta)$, condutividade hidráulica na capacidade de campo (Kcc) e potencial mátrico $\left(\phi_{m}\right)$ nas diferentes profundidades. 
de campo variando de 0,006 a 0,008 MPa para o Latossolo Roxo, maiores que os encontrados nas camadas do Bw da TR. A umidade volumétrica no ponto de murchamento permanente $\left(\theta_{\mathrm{PMP}}\right)$ apresentou uma relação estreita com a morfologia do solo e foi sempre menor no horizonte Bw comparado ao Bt (Figura 6).

Os valores de condutividade hidráulica na capacidade de campo (KCC) foram menores no horizonte Bt que no horizonte Bw (Figura 6). Os val ores obtidos apresentaram, no entanto, diferenças de menos de uma ordem de grandeza $(\bar{x}=0,0037$; CV $=69 \%$ ) para uma amplitude de variação absoluta de $12,6 \%$ na umidade volumétrica nas diversas profundidades do solo. Saunders et al. (1978) encontraram para uma variação de umidade volumétrica de $6,4 \%$ uma variação na condutividade hidráulica de aproximadamente 100 vezes.

\section{CONCLUSÕES}

1. A Terra Roxa Estruturada Latossólica apresentou um horizonte Bt, cujas características físicas (mai or densidade e microporosidade) e morfológicas (estrutura em blocos e cerosidade) determinaram maiores valores de capacidade de campo $(\theta \mathrm{cc})$, ponto de murchamento permanente $\left(\theta_{\mathrm{PMP}}\right)$, potencial mátrico $\left(\phi_{\mathrm{m}}\right)$ e condutividade hidráulica na capacidade de campo (Kcc) quando comparados aos do horizonte Bw.

2. O método- $\theta$ (Libardi et al., 1980) ajustou com al to coeficiente de correlação os val ores de umidade volumétrica no processo de redistribuição de água. A suposição de gradiente do potencial hidráulico unitário ocorreu somente para o horizonte Bw. O horizonte Bt apresentou valores de gradiente do potencial hidráulico maiores que um. Tais variações nos valores de gradiente do potencial hidráulico determinaram uma superestimativa nos val ores de condutividade hidráulica do solo no Bt, quando comparados com os do método de Hillel et al. (1972). As variações no valor do gradiente de potencial hidráulico podem ser utilizadas na identificação de diferenças morfológicas, físicas ehídricas num perfil de solo.

\section{LITERATURA CITADA}

ARRUDA, F.B.; ZULLO J r., J . \& OLIVEIRA, J.B. Parâmetros de solo para cálculo da água disponível com base na textura do solo. R. Bras. Ci. Solo, 11:11-15, 1987.

BAKER, F.G.; VENEMAN, P.L.M. \& BOUMA, J . Limitations of the instantaneous profile method for field measurement of insaturated hydraulic conductivity. Soil Sci. Soc. Am. Proc., 38:885-888, 1974.
DEMATTÊ, J .L.I \& \& MARCONI, A. A drenagem na mineralogia de sol os desenvolvidos de diabásio em Piracicaba (SP). R. Bras. Ci. Solo, 15:1-8, 1991.

DEMATTÊ, J.L.I.; MARCONI, A.; SPAROVEK, G. \& VIDAL TORRADO, P. Estimativa da evolução do intemperismo mediante ganhos e perdas de ions numa sequência de sol os desenvolvidos de diabásio e influenciados pela drenagem em Piracicaba (SP). R. Bras. Ci. Solo, 15:69-73, 1994.

EMPRESA BRASILEIRA DE PESQUISA AGROPECUÁRIA EMBRAPA. Serviço Nacional de Levantamento e Conservação do Solo. Manual de métodos de análise de solo. Rio de J aneiro, 1979. não paginado.

EMPRESA BRASILEIRA DE PESQUISA AGROPECUÁRIA EMBRAPA. Serviço Nacional de Levantamento e Conservação do Solo. Instituto Agronômico do Paraná. Levantamento de Reconhecimento dos Solos do estado do Paraná. Curitiba, 1984. 791p.

FREIRE, J .C. Condutividade hidráulica e capacidade de campo de Latossolo Roxo distrófico não saturado. R. Bras. Ci. Solo, 3:73-77, 1979.

HAISE, H.R.; HAAS, H.J . \& J ENSEN, L.R. Soil moisturestudies of some great plains soils. II. Field capacity related to $1 / 3$ atmosphere percentage and minimum point related to 15 and 26 atmosphere percentages. Soil Sci. Soc. Am. Proc., 10:20-25, 1955.

HILLEL, D.; KRENTOS, V.O. \& STYLIANOU, Y. Procedureand test of an internal drainage method for measuring soil hydraulic characteristics in situ. Soil Sci., 114:395-400, 1972.

LAUREN,J .G.; WAGENET, R.J .; BOUMA, J \& WOSTEN, J .H.M. Variability of saturated hydraulic conductivity in a Glossaquic Hapludalf with macopores. Soil. Sci., 145:2028, 1988.

LEMOS, R.C. \& SANTOS, R.D. Manual de descrição e coleta de solo no campo. 2.ed., Campinas, Sociedade Brasileira de Ciência do Solo, Serviço Nacional de Levantamento e Conservação do Solo, 1984. 56p.

LIBARDI, P.L. Dinâmica da água no solo. Piracicaba, Edição do Autor, 1995. 497p.

LIBARDI, P.L.; REICHARDT, K.; NIELSEN, D.R. \& BIGGAR, J.W. Simple field methods for estimating soil hydraulic conductivity. Soil Sci. Soc. Am. J ., 44:3-7, 1980.

MONIZ, A.C. Evolução de conceitos no estudo da gênese de solos. R. Bras. Ci. Solo, 20:349-362, 1996.

MONIZ, A.C.\& BUOL, S.W. Formation of an oxisol-ultisol transition in São Paulo-Brazil. I. double-water flow model of soil development. Soil Sci. Soc. Am. J ., 46:1228-1233, 1982.

MONIZ, A.C.; BUOL, S.W. \& WEED, S.B. Formation of an oxisolultisol transition in São Paulo-Brazil. II. Iateral flow dynamics of chemical weathering. Soil Sci. Soc. Am. J., 46:1234-1239, 1982.

MONIZ, A.C.; MANFREDINI, S. \& DEMATTÊ, J .L.I . Variações morfológicas, mineralógicas e hídricas em terra roxa estruturada ao longo de uma vertente em Rio das Pedras. R. Bras. Ci. Solo, 18:513-520, 1994.

REICHARDT, K. Processos de transferência no sistema soloplanta-atmosfera. 4.ed. Campinas, Fundação Cargill, 1985. $446 p$. 
REICHARDT, K. \& LIBARDI, P.L. An analysis of soil water movement in the field. In: HIDROLOGICAL field site characterization. Piracicaba, CENA-ESALQ, 1974. 21p. (Boletim Científico, 21)

SAUNDERS, L.C.V; LIBARDI, P.L. \& REICHARDT, K. Condutividade hidráulica da Terra Roxa Estruturada em condições de campo. R. Bras. Ci. Solo, 3:137-140, 1978.

SOUZA, M.L.P.; LIBARDI, P.L. \& REICHARDT, K. Propagação de erros na medida de fluxos de água no solo sob condições de campo. R. Bras. Ci. Solo, 3:137-140, 1979.
UEHARA, G. \& GILLMAN, G. The mineralogy, chemistry and physics of tropical soils with variablecharge clays. Boulder, Westwiew Tropical Agriculture Series, 1981. 170p.

VAUCLIN, M.; IMBERNON, J.\& VACHAUD, G. Analyse comparative de different methods de determination de la conductivité hydraulique des sols non saturés de la zone centre-nord du Senégal. Agron. Trop., 38:186-197, 1982.

ZIMBACK, C.R.L. \& CARVALHO, A.M. Caracterização físico hídrica dos solos de três bacias hidrográficas na região de Marília-SP. R. Bras. Ci. Solo, 20:459-466, 1996. 